\title{
Multiple Myeloma: a Retrospective Analysis of 61 Patients from a Tertiary Care Center
}

\author{
Sadia Sultan ${ }^{1 *}$, Syed Mohammed Irfan ${ }^{1}$, Saira Parveen ${ }^{1}$, Hamza Ali ${ }^{2}$, Maria \\ Basharat $^{2}$
}

\begin{abstract}
Background: Multiple myeloma (MM) is an acquired clonal B-cell malignancy which primarily affects elderly individuals with an annual incidence of approximately $1 \%$ of all malignancies. Our aim is to study demographic and clinicopathological features of adult Pakistani MM patients at presentation. Materials and Methods: This single centre retrospective study extended from January 2010 to December 2014. Data were retrieved from the patients' maintained records on predetermined performa. Results: Overall, 61 patients were diagnosed at our institution with MM during the study period. There were 43 males and 18 females. Age ranged between 34 and 81 years with a mean of $\mathbf{5 6 . 1} \mathbf{1 2 . 8}$ and a median of 57 years. The male to female ratio was $\sim 2: 1$. Common presenting complaints included fatigue $(81.9 \%)$, backache $(80.3 \%)$ and bone pain $(67.2 \%)$. Physical findings revealed pallor $(44.2 \%)$ as a presenting clinical feature. The mean hemoglobin value was $8.9 \pm 1.7 \mathrm{~g} / \mathrm{dl}$ with a mean MCV of $85.3 \pm 11.0 \mathrm{fl}$. Severe anemia with hemoglobin $<8.5 \mathrm{gm} / \mathrm{dl}$ was seen in $40.9 \%$. The mean total leukocyte count was $8.9 \pm 8.2 \times 10^{9} / 1$, the ANC was $5.0 \pm 3.1 \times 10^{9} / 1$ and the mean platelet count was $188.4 \pm 150.6 \times 10^{9} / 1$. Conclusions: MM in Pakistani patients is seen in a relatively young population with male preponderance. The majority of patients present with symptomatic anemia and backache to seek medical attention. However, clinico-pathological features appear comparable to the published literature.
\end{abstract}

Keywords: Multiple myeloma - demographic data - Pakistan.

Asian Pac J Cancer Prev, 17 (4), 1833-1835

\section{Introduction}

Symptomatic multiple myeloma (MM) is a clonal malignant neoplasm of plasma cells originating in the bone marrow with the median survival of $\sim 4$ years (Sun et al., 2015; Landgren et al., 2009). As per United States surveillance and epidemiology multiple myeloma accounts for $1 \%$ of overall malignancies and approximate $10 \%$ of all hematopoietic malignancies (Rajkumar., 2011; Vincent., 2014). Overall incidence of myeloma increases with age. Median age at diagnosis is around 65-70 years (Mehdi et al., 2013; Wang et al., 2015). Multiple myeloma is slightly more common in men than in women.

Certain risk factors have been proposed as etiologically causative, including radiation, chemicals such as asbestos, benzene, arsenic, carbon monoxide; agricultural possibly related to exposure to pesticides, recurrent infections leading to chronic antigenic stimulation in various illnesses and lastly genetic factors (Eriksson et al., 1992). Relationship of myeloma with these risk factors, however, has been still conflicting.

Myeloma tumor cells release certain factors which activate the osteoclastic activity resulting in bone resorption, hypercalcemia, bony lytic lesions, bone pains and eventually pathological fractures (Sutandyo et al., 2015). Renal involvement occurs frequently and is due to deleterious effects of light chains on renal tubular epithelium being associated with poor prognosis. CRAB clinical manifestation includes raised calcium, renal impairment, anemia and bone lytic lesions are another frequent finding in these symptomatic myeloma patients. Clinical course of MM is regularly bi-phasic with an initially persistent stable chronic phase followed by an accelerated terminal phase.

Most of the data on myeloma are from Western countries. There are no incidence figures of MM available in Pakistan. Due to paucity of data from developing countries, we reviewed our experience with multiple myeloma in Pakistan. We evaluated clinical characteristics, and laboratory data at diagnosis to establish our own local facts.

\section{Materials and Methods}

This is a retrospective descriptive study conducted from January 2010 till December 2014. Detailed 
information available for 61 patients with multiple myeloma were enrolled in the study. Socio-demographic data including age and gender were recorded. All patients underwent detailed history, general physical and systemic examination.

Diagnosis of MM was established by World Health Organization (WHO) criteria (McKenna et al., 2008). Diagnosis required meeting three criteria's includes

i). M-protein in serum or urine, $i i$ ). Bone morrow clonal plasma cells, iii). Related organ or tissue impairment

Patients with other lymphoid neoplasm (both B and T-cell lineages) were excluded. Patients with another associated malignancy or having relapsed/ refractory MM were also excluded.

Peripheral venous blood samples were taken for complete blood count and hematological parameters were determined by Automated Cell Dyne Ruby. Serum creatinine and calcium were detected by HITACHI 912 (Japan) by photometric assay. M-Proteins were determined by serum protein electrophoresis which was done by Interlab Genio-S instrument. Bone marrow aspirate and trephine biopsy specimen were taken from posterior iliac crest with Jamshidi needle. Each bone marrow aspirations and biopsies were reviewed by consultant hematologists.

\section{Statistical analysis}

Statistical analysis was carried out using SPSS version 22. Qualitative data were presented in terms of frequencies and percentages. Mean and standard deviation were reported for quantitative variables.

\section{Results}

\section{Demographical outline}

During the study period, 61 patients were diagnosed as Multiple Myeloma. Out of 61 patients, 43 were males (70.4\%) and 18 were females (29.5\%) with male to female ratio of was $\sim 2: 1$. Age ranged between 34 and 81 years with a mean age of $56.05 \pm 12.8$ years and median age of 57 years. Age stratification is shown in table- 1 .

\section{Clinical findings}

The common presenting complaints included fatigue in $50(81.9 \%)$ patients, backache in $49(80.3 \%)$ patients and bone pain in $41(67.2 \%)$ patients. None of our patient presented with thrombotic manifestation, however 12 (19.6\%) patients presented with sign and symptoms of hyperviscosity (blurring of vision, headache, dizziness, muscular weakness). Physical examination revealed pallor as a predominant finding detected in 27 (44.2\%) patients followed by petechial and purpural rashes in 10 (16.3\%) patients.

\section{Laboratory profile}

The mean hemoglobin levels were $8.9 \pm 1.7 \mathrm{~g} / \mathrm{dl}$ $(3.2-13.3 \mathrm{gm} / \mathrm{dl})$ with the mean MCV of $85.3 \pm 11.0$ fl $(69-105 \mathrm{fl})$. The mean total leukocyte count of $8.9 \pm 8.2 \times 10^{9} / 1\left(0.3-31.3 \times 10^{9} / 1\right)$; absolute neutrophils count of $5.0 \pm 3.1 \times 10^{9} / 1\left(0.2-12.2 \times 10^{9} / 1\right)$ and the mean platelets count were $188.4 \pm 150.6 \times 10^{9} / 1\left(9-851 \times 10^{9} / 1\right)$.

Anemia $(\mathrm{Hb}<10 \mathrm{gm} / \mathrm{dl})$ was appreciated in $39(63.9 \%)$
Table 1. Age Stratification in Patients with Multiple Myeloma from Pakistan

\begin{tabular}{ccccc}
\hline \multirow{2}{*}{ Age groups } & Male & Female & Total & Percentage \\
\cline { 2 - 5 } & $\mathrm{n}=43$ & $\mathrm{n}=18$ & $\mathrm{n}=61$ & $\%$ \\
\hline$<40$ & 5 & 3 & 8 & 13.1 \\
$40-60$ & 24 & 9 & 33 & 54 \\
$>60$ & 14 & 6 & 20 & 32.7 \\
\hline
\end{tabular}

patients. Severe anemia $(\mathrm{Hb}<8.5 \mathrm{gm} / \mathrm{dl})$ was noted in 25 $(40.9 \%)$ patients. Thrombocytopenia (platelets count $\left.<100 \times 10^{9} / 1\right)$ was detected in $14(22.9 \%)$ patients, while severe thrombocytopenia (platelets $<20 \times 10^{9} / 1$ ) was seen in $3(4.9 \%)$ patients.

Of the 61 study patients, $29(47.5 \%)$ had hypercalcemia (S.Ca $>12 \mathrm{mg} / \mathrm{dl}$ ) while renal impairment (serum creatinine $>1.5 \mathrm{mg} / \mathrm{dl}$ ) was noted in $25(40.9 \%)$ patients. Radiological survey showed different levels of skeletal involvement in $48(78.6 \%)$ of patients.

\section{Discussion}

Multiple myeloma (MM) is a plasma cell dyscarsia, characterized by bone marrow infiltration with clonal plasma cells, production of monoclonal immunoglobulin (paraprotein), and associated with end organ damage including lytic lesions in the bones, renal impairment, hypercalcemia and anemia. MM is incurable disease with the median patient survival of 3-4 years (Sashidharan et al., 2015).

This hematological disorder is not uncommon world wide. There is a handful studies reported from Pakistan on this hematopoietic malignancy (Shaheen et al., 1999; Mansoor et al., 2005; Inamullah et al, 2010; Basit et al., 2014). The present study has demonstrated demographic, clinical features and hematological markers in Pakistani MM patients.

MM usually develops insidiously and is mainly observed in people over the age of 60 years with male predominance. The ages of the patients included in the study group ranged from 34-81 years and most of patients presented in the 5 th decade of life, with the mean age of 56 years. It was noted that the mean age and gender distribution in our patients were more or less similar with that were reported (59 and 61 years) in the previous local studies (Mansoor et al., 2007; Basit et al., 2014). Similarly a regional study reported from India, showed the mean age of 58.8 years in their MM patients (Kaur et al., 2014). Recent one Nigerian study reported the median age of 58 years, in concurrence to our findings (Omoti et al., 2012).

In the developed world, the median age for disease presentation is reasonably high. One recent Swedish study reported the median age of 72 years in western myeloma patients (Mohammadi et al., 2015). The plausible elucidations are varied genetic composition and more accountable is the higher mean age in western countries compared with South Asian vicinity.

In general, our patients presented with the more advanced disease with frequent complications. Our majority of patients presented with fatigue $(81.9 \%)$ and backaches $(80.3 \%)$. Comparable presenting complaints 
were observed by prior Pakistani and USA studies, detected fatigue and backaches in $78 \%$ and $80 \%$ respectively (Shaheen et al., 1999; Kyle et al., 2003). Bony pains and easy fatigability were also the most common symptoms in another study reported from India by Subramanian (Subramanian et al., 2009).

Primarily disease manifestations are due to the infiltration by plasma cells and secretion of M-protein by malignant clones. The most frequent clinical characteristic of MM is anemia. A hemoglobin concentration of $<12 \mathrm{~g} / \mathrm{dl}$ detected in $73 \%$ of patients at disease presentation which frequently contributes to easy fatigability and weakness in approximately $82 \%$ of patients (Kaur et al., 2014). Anemia $(\mathrm{Hb}<10 \mathrm{gm} / \mathrm{dl})$ was also a common presenting feature $(63.9 \%)$ in our series. Clinical features in our study somewhat correlated with the study conducted by Inamullah and Yassin showing that symptomatic anemia as a principal laboratory finding (Inamullah et al., 2010; Yassin et al., 2013).

Approximately $75 \%$ of MM patients had reported bone lesions included punched-out lytic lesions, osteoporosis or fractures on conventional radiography (Kaur et al., 2014). Analogous to this, radiological survey showed different levels of skeletal involvement in 48 (78.6\%) of our patients.

In the present study, raised serum creatinine levels and hypercalcemia were found in $40.9 \%$ and $47.5 \%$ of the patients respectively where these results were compared with Mansoor et al, showed that $51.2 \%$ of their patients had hypercalcemia (Mansoor et al., 2005). Previously, one large regional study reported hypercalcemia and renal derangement in $23 \%$ and $43 \%$ of patients respectively (Shaheen et al., 1999).

We acknowledge limitations of our study, including its retrospective nature and relatively small sample study. Another limitation was the unavailability of data concerning the stage of disease.

In conclusion, multiple myeloma is a disease with a variable clinical presentation with multiple system involvement. Younger age of disease onset is some notable features of myeloma in Pakistan. Clinico-pathological features are appearing comparable to published data. Fatigability associated with bone pains is the commonest presentation. MM should be considered as a differential in the workup of anemia in patients above 60 years age.

\section{References}

Basit A, Siddiqui N, Hameed A, et al (2014). Factors affecting outcome of patients with multiple myeloma. J Ayub Med Coll Abottabad, 26, 376-9.

Eriksson M, Karisson M (1992). Occupational and other environ mental factors and multiple niycolma: a population based case-control study. Brl, Ind Med, 49, 95-103.

Inam ullah, Raziq F, Tahir M, Wazir R, Rafiq A (2010). Hematological Presentation of Multiple Myeloma in Khyber Pakhtunkhwa. Gomal J Med Sciences, 8, 130-3.

Kaur P, Shah BS, Baja P (2014). Multiple myeloma: a clinical and pathological profile. Gulf J Oncolog, 1, 14-20.

Kyle RA, Gertz MA, Witzig TE, et al (2003). Review of 1027 patients with newly diagnosed multiple myeloma. Mayo Clinic Proc, 78, 21-33.
Landgren O, Weiss BM (2009). Patterns of monoclonal gammopathy of undetermined significance and multiple myeloma in various ethnic/racial groups: support for genetic factors in pathogenesis. Leukemia, 23, 1691-7.

McKenna RW, Kyle RA, Kuehl WM, et al (2008). WHO classification of tumours of haemopoietic and lymphoid tissues. Lyon: International Agency Res Cancer, 202.

Mehdi WA, Zainulabdeen JA, Mehde AA (2013). Investigation of the antioxidant status in multiple myeloma patients: effects of therapy. Asian Pac J Cancer Prev, 14, 3663-7.

Mansoor S, Siddiqui I, Adil S, Kakapeto GN, Fatmi Z (2005). Frequency of hypercalcemia in patients of multiple myeloma in Karachi. J Coll Physicians Surg Pak, 15, 409-12.

Mansoor S, Siddiqui I, Adil S, et al (2007). Anion gap among patients of multiple myeloma and normal individuals. Clin Biochem, 40, 226-9.

Mohammadi M, Cao Y, Glimelius I, et al (2015) .The impact of comorbid disease history on all-cause and cancer-specific mortality in myeloid leukemia and myeloma - a Swedish population-based study. BMC Cancer, $\mathbf{1 5}, 850$.

Omoti CE, Nwannadi AI, Obieche JC, Olu-Eddo AN (2012). The epidemiological features of lymphoid malignancies in Benin City, Nigeria: a 15 years study. Pan Afr Med J,11, 10.

Rajkumar SV (2011). Treatment of multiple myeloma. Nat Rev Clin Oncol, 8, 479-91.

Shaheen H, Ghanghroo I, Malik I (1999). Clinicopathological features and management of Pakistani patients with multiple myeloma. J Pak Med Assoc, 4, 233-7.

Sashidharan N, Shenoy S, Kishore MK, Thanusubramanian H (2015). Comparison of two therapeutic regimes, lenalidomide with dexamethasone and thalidomide with dexamethasone, in the treatment of multiple myeloma at a tertiary care hospital in India. J Clin Diagn Res, 9, 1-4.

Sun JJ, Zhang C, Zhou J, Yang HL (2015). Pooled analysis of pomalidomide for treating patients with multiple myeloma. Asian Pac J Cancer Prev, 16, 3163-6.

Sutandyo N, Firna E, Agustina J, et al (2015). Clinicopathology profile and bone involvement of multiple myeloma patients in dharmais national cancer hospital, indonesia. Asian Pac J Cancer Prev, 16, 6261-5.

Subramanian R, Basu D, Dutta TK (2009). Prognostic significance of bone marrow histology in multiple myeloma. Indian J Cancer, 46, 40-5.

Vincent Rajkumar S (2014). Multiple myeloma: 2014 Update on diagnosis, risk-stratification, and management. Am J Hematol, 89, 999-1009.

Wang L, Wang KF, Chang BY, Chen XQ, Xia ZJ (2015). Once-weekly subcutaneous administration of bortezomib in patients with multiple myeloma. Asian Pac J Cancer Prev, 16, 2093-8.

Yassin AK (2013). Clinical and Laboratory Profiles of 109 Patients diagnosed as Multiple Myeloma in Erbil City. $J$ Fac Med Baghdad, 55, 121-4. 